ultraviolet radiation. Such scattered photons are again likely to be highly linearly polarised. By arguments similar to those above it is clear that the net electric vector of the scattered photons would tend to be aligned with the rotation axis of the binary system. The amount of such polarised scattered $\mathrm{X}$ rays received would vary depending on how much of that part of the star that is illuminated by the X-ray source is visible - that is, it would vary with the orbital period. In addition, the smaller the angle between our line of sight and the rotation axis of the binary system, the greater the amount of time for which the illuminated part of the star is visible and hence the smaller the size of the variation. Thus, in principle, such an observation could tell us the inclination of the binary orbit to our line of sight. Because such a small fraction of the emitted $X$ rays actually strikes the companion star, such an effect would, however, be difficult to detect.

$\mathrm{X}$-ray polarimetry is at present in its infancy, bui it seems likely to grow to maturity over the next few years. In the past, the best-known argument for $\mathrm{X}$-ray polarimetry has been that it is often symptomatic of non-thermal synchrotron-type emission. Drs Lightman, Shapiro and Rees have drawn attention to other sources of X-ray polarisation and, more importantly, have shown how X-ray polarimetry can be used as a testing ground for theoretical models, and as a means of probing the structure, of the binary X-ray stars.

\section{Ozone over Britain}

\section{from Peter D. Moore}

IT is no longer possible for the British to be complacent about ozone pollution. Though Los Angeles is still the worst hit area of the world, having occasional peaks of 70 p.p.h.m. of ozone in its air (Marx, Science, 187, 731 ; 1975) and having at least once reached 99 p.p.h.m., the problem is far more widespread: even in Melbourne peaks of 28 p.p.h.m. have recently been recorded.

In the United States, ozone is regarded as by far the most damaging of air pollutants to plants. In New Jersey, Pell and Brennan (Environ. Pollut., 8, 23; 1975) consider that $\$ 128,000$ worth of damage was caused to agricultural and ornamental plants in 1972 , and estimated that losses of over $\$ 35,000,000$ have been made for California. Ozone is not yet considered an economic threat to British agriculture.

In 1972, Atkins et al. (Nature, 235,
372 ; 1972) demonstrated the production of atmospheric ozone over southern England and later Derwent and Stewart (Nature, 241, 342; 1973) published data for central London in which they found daily peaks of $8-12$ p.p.h.m. Recently Cox et al. (Nature, 255, 118; 1975) have concluded that a proportion of southern England's ozone may have been transported considerable distances, of the order of $100-1,000 \mathrm{~km}$. It seems clear, therefore, that Britain has an ozone pollution problem, though it is not entirely of her own making.

Bell and Cox (Environ. Pollut., 8, $163 ; 1975$ ) have now published data, obtained in 1972, concerning plant damage by ozone in Britain. They exposed the ozone-sensitive variety of Bel-W3 of tobacco to the atmosphere in Ascot (32 km west of central London) and at the same time monitored the air for ozone. They observed the characteristic symptoms of ozone toxicity (necrotic spots on the leaf surface) and their atmospheric measurements showed that ozone levels had risen above 4 p.p.h.m. on 322 occasions between July 4 and September 15 and above 6 p.p.h.m. on 83 occasions. On one occasion a level of 10 p.p.h.m. was maintained for $5 \mathrm{~h}$. (The United States air quality standard of the Environmental Protection Agency is 8 p.p.h.m. for one hour.) Bell and Cox confirmed that there was a strong positive correlation between the degree of leaf damage and the length of time for which each test plant had been exposed to ozone levels in excess of 4 p.p.h.m. More resistant tobacco varieties showed very low levels of damage and no significant correlation with ozone exposure; no correlation at all was demonstrable with sulphur dioxide concentration.

These results show that diurnal peaks in ozone, at levels which could be injurious to both man and plants, are not restricted to central London, but could be occurring over a much wider radius. No records have yet been reported of ozone damage to crops or to wild plants in Britain but, as Bell and Cox point out, growth may be suppressed without symptoms being visible.

Recent experiments on a number of herbaceous plants in America, however, by Harward and Treshow (Environ. Cons., 2, 17; 1975) suggest that visible symptoms and growth reduction are rare at levels of 5-7 p.p.h.m. ozone for about $2 \mathrm{~h}$ per day, but become apparent in some species when levels of 15 p.p.h.m. are used. In August 1973, levels as high as 17 p.p.h.m. were recorded at Harwell, $80 \mathrm{~km}$ west of London, so perhaps visible damage will soon make itself apparent and we can recognise that we do indeed have an ozone problem.

\section{Agglutination of tumour cells}

from Robert Shields

Much research effort has gone into investigations of why plant lectins often agglutinate tumour cells to a greater extent than their normal counterparts. As with all generalisations about tumour cells, enhanced agglutination is not an invariant property of transformation. It is, however, sufficiently widespread in the model systems used by cell biologists to have promoted intensive study.

Using the lectin concanavalin A (Con A) it has been shown that agglutinability of some lines of cells is directly related to their tumorigenicity when injected into animals (Inbar et al., Nature new Biol., 236, 3; 1972). Also, cells transformed with temperature-sensitive tumour viruses exhibit high agglutinability at the permissive temperature (where the transformed phenotype is expressed) and lowered agglutinability at the non-permissive temperature (Burger and Martin, Nature new Biol., 237, 9; 1972; Eckhart et al., Proc. natn. Acad. Sci. U.S.A., 68, 283; 1971). The experiments show that high agglutinability is closely associated with oncogenic transformation. This idea was considerably strengthened by experiments that used a variety of selective pressures to produce revertants of tumour cells with approximately normal behaviour. The revertants are less agglutinable and less tumorigenic than their transformed parents (Pollack, In Vitro, 6, 58; 1970; Pollack et al., Proc. natn. Acad. Sci. U.S.A., 60, 126; 1968). One of the best pieces of evidence for the close linkage of agglutinability and transformation is from work carried out by two separate groups (Culp and Black, J. Virol., 9, 611; 1972; Ozanne and Sambrook, Lepetit Colloq., 2, 248; 1971) on revertants. They used the fact that agglutination of tumour cells with high doses of Con A leads to cell death. After treating transformed cells with high concentrations of the lectin and isolating surviving cells, they were able to establish cell lines with reduced agglutinability and a more normal phenotype.

It has recently been suggested that tumour cells are highly agglutinable because they have more microvilli on their surfaces and that the presence of these microvilli is regulated by cyclic AMP (Willingham and Pastan, Proc. natn. Acad. Sci. U.S.A., 72, 1263; 1975). These workers suggest that microvilli help agglutination by bringing larger areas of cells' surfaces into close apposition, allowing them to be cross-linked by lectins. They suggest that it is the low level of cyclic AMP 